\title{
Model for knowledge-sharing strategies: a game theory analysis
}

\author{
S. P. $\mathrm{HO}^{1 *}, \mathrm{Y} . \mathrm{HSU}^{2}$ and E. $\mathrm{LIN}^{3}$ \\ ${ }^{1}$ Department of Civil Engineering, National Taiwan University, Taipei 10617, Taiwan, Republic of China \\ ${ }^{2}$ Department of International Business, National Taiwan University, Taipei 10617, Taiwan, Republic of China \\ ${ }^{3}$ Department of Civil Engineering, National Taiwan University, Taiwan, Republic of China
}

Received 6 December 2010; accepted 9 December 2010

\begin{abstract}
Recently, knowledge management $(\mathrm{KM})$ has drawn immense attention from researchers and practitioners in many industries and has become increasingly important in business management practice. From the resource-based view, the integration of individuals' specialized organizational capability through KM has been considered crucial to the creation and sustainability of competitive advantages. However, most KM literature tends to focus on the 'technology side' of KM such as issues in information and communication technology (ICT) platforms or systems. As we have observed, many KM attempts died with their costly, advanced, high-tech ICT systems. It is becoming clear that KM cannot be successful without appropriate strategies for encouraging the sharing of valuable knowledge, because people in organizations may not be motivated to share knowledge and may even prefer not to share their knowledge in order to preserve their intellectual or proprietary values in organizations. This paper aims to study the behavioural dynamics of knowledge-sharing in organizations and to derive a model for knowledge-sharing strategies. Two fundamental strategic choices for firms studied in this paper are whether or not to invest in ICT platforms and whether or not to offer rewards for sharing. A theoretic model based on game theory is developed to study the interaction between employees' knowledgesharing behaviours and firms' knowledge-sharing strategies. Then, based on the results of game modelling, a new knowledge taxonomy is developed from the perspective of knowledge-sharing dynamics and a framework for knowledge-sharing strategies is proposed.
\end{abstract}

Keywords: Game theory, knowledge management, knowledge-sharing, organization, strategy.

\section{Introduction}

Knowledge has emerged as the most strategically significant resource of the firm, and the integration of individuals' specialized organizational capability is crucial to the creation and sustainability of competitive advantages (Grant, 1996). From the resource-based view (RBV), knowledge management (KM) can be considered one of the most critical processes for business success in today's intensively competitive environment. Many firms and organizations have embraced the KM philosophy and adopted various management practices and strategies. However, for those who practice $\mathrm{KM}$, the major efforts in $\mathrm{KM}$ implementation are to develop the required information and communication technology (ICT) platforms. As we have observed, many $\mathrm{KM}$ attempts ended with their costly and advanced high-tech ICT platforms. In fact, it is becoming clear that KM cannot be successful without appropriate strategies for encouraging the sharing of knowledge, because individuals may not be motivated to share valuable knowledge and may even prefer not to share their knowledge in order to preserve their intellectual or proprietary values in organizations. As argued by Cabrera and Cabrera (2002), knowledge-sharing can also fall into the trap of social dilemma, under which the individuals could be better off by being free-riders than being the ones who share. Without the premise of individuals' willingness to share useful knowledge, there will be no valuable inputs for any KM systems.

With the ICT platforms, knowledge can be disseminated corporate-wide and can be stored for repeated

*Author for correspondence. E-mail: spingho@ntu.edu.tw 
usage. The ICT platforms may also include communication platforms for informal or social interactions which are suitable for sharing tacit knowledge. Although the ICT platforms may lower the costs of obtaining knowledge, we argue that the ICT platforms have limited advantages in reducing the costs of sharing. For example, the time and efforts to summarize lessons learned from a project have little to do with ICT platforms and are not likely to be reduced by the platforms. This is also why many KM attempts fail after expensive investments in ICT platforms. Firms naively believe that employees will begin to or be motivated to share knowledge with the implementation of ICT platforms. Therefore, it is of strategic importance for firms to decide whether or not to invest in ICT platforms as a part of a KM system and to rethink what motivates the sharing of valuable knowledge.

An instrument often used to facilitate knowledgesharing is the offer of monetary rewards. 'Good knowledge should be purchased' (Moon and Park, 2002). Many firms thus focus on developing and designing the reward and punishment systems to facilitate knowledge-sharing by the employees in organizations (Hendriks, 1999). For example, firms can record the sharing activities or measure the value of the knowledge and offer rewards commensurate with the sharing activities and/or the contribution of the shared knowledge. Sufficient rewards can compensate the employees' costs of sharing, and thus encourage employees to share their knowledge. In addition to monetary rewards, rewards may also include non-monetary rewards, such as esteem, praise and social recognition, which can be very effective in fostering enduring attitudes towards knowledge-sharing. However, since monetary rewards can result in significant costs to firms, this study explicitly focuses on the impacts of monetary rewards and considers whether or not to offer monetary rewards as another strategically important issue in facilitating knowledge-sharing.

This paper studies the behavioural dynamics of knowledge-sharing in firms and derives strategies for facilitating the sharing of knowledge. First, a theoretic model based on game theory is developed to solve for the conditions that determine the knowledge-sharing behaviours of employees. Since the sharing of knowledge relates to the competitive and cooperative relationships between employees and firms, game theory is considered as a natural method to analyse the knowledge-sharing problem. Second, based on the model, we discuss how to categorize knowledge from the perspective of knowledge-sharing dynamics. Lastly, based on the new knowledge taxonomy from the game theory analysis, a framework consisting of three hypotheses is proposed for the strategies of knowledge-sharing.

\section{Methodology: game theory analysis}

Game theory can be defined as the study of mathematical models of conflict and cooperation between intelligent, rational decision-makers. There are two basic types of games based on the timing of decisionmaking: static games and dynamic games. In a static game, the players act simultaneously, meaning that each player chooses his/her action without knowing that of others. On the contrary, in a dynamic game, the players act sequentially and observe other players' actions in previous moves. Because individuals make decisions on sharing knowledge after observing the actions taken by the firm, the dynamic game will be used for modeling and analysis in this study.

It is intuitive to represent a dynamic game by a tree-like structure, also called the 'extensive form' representation. We will use the following simplified Market Entry example to demonstrate the concepts of a game analysis. A new firm, New Inc., wants to enter a market to compete with a monopoly firm, Old Inc. The monopoly firm does not want the new firm to enter the market, because the new entry will reduce the incumbent firm's profits. Therefore, Old Inc. threatens New Inc. with a price war if New Inc. enters the market. Figure 1 shows the extensive form of the market entry game. If the payoffs shown in Figure 1 are known to all players, the payoffs are 'common knowledge' to all players. In addition, the players of a game are assumed to be rational; i.e. it is assumed that the players will always try to maximize their payoffs. If the players' rationality and the game structure, including payoffs, are common knowledge, the game is called a game of 'complete information.' The game tree shows (1) New Inc. chooses to enter the market or not, and then Old Inc. chooses to start a price war or not, and (2) the payoff of each decision combination.

To answer how each player will play/behave in this game, we introduce the concept of the 'Nash equilibrium', one of the most important concepts in game theory. The Nash equilibrium is a set of actions that will be chosen by each player. In other words, in a

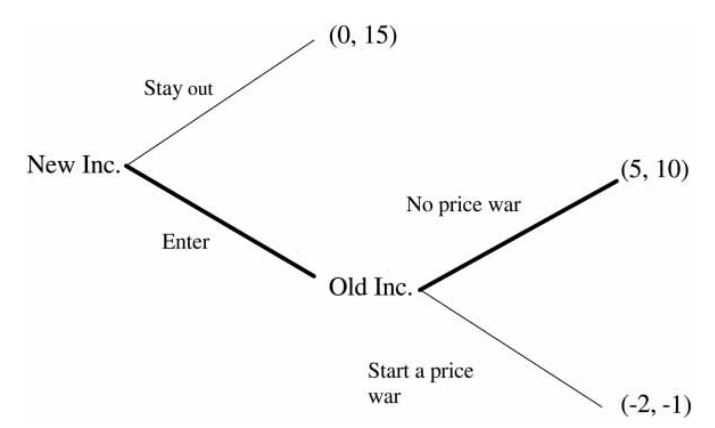

Figure 1 Simplified market entry game 
Nash equilibrium, each player's strategy should be the best response to the other player's strategy, and no player wants to deviate from the equilibrium solution. Thus, the equilibrium or solution is 'strategically stable' or 'self-enforcing' (Gibbons, 1992). In a dynamic game, the Nash equilibrium is a subgame perfect Nash equilibrium, which satisfies the sequential rationality required for the solution of a dynamic game.

An intuitive conjecture of the solution of the Market Entry game is that New Inc. will 'stay out' because Old Inc. threatens to 'start a price war' if New Inc. plays 'enter'. However, Figure 1 shows that the threat to start a price war is not credible because Old Inc. can only be worse off by starting a price war if New Inc. does enter. On the other hand, New Inc. knows the incredibility of the threat, and therefore will maximize the payoff by playing 'enter'. As a result, the subgame perfect Nash equilibrium of the market entry game is (enter, no price war if enter) a strategically stable solution that does not rely on the player to carry out an incredible threat. In a dynamic game, the Nash equilibrium is a subgame perfect Nash equilibrium, which satisfies the sequential rationality by maximizing each player's payoffs in the subgames backward recursively. We shall apply this technique in solving the knowledge-sharing game.

\section{Game theorectic analysis of knowledge- sharing dynamics}

\section{Model assumptions}

In the model, firms choose the strategies for knowledgesharing and then employees decide whether or not to share knowledge. We assume that employees are rational individuals who decide to 'Share' or 'Not share' based on their total payoffs from sharing. For firms, we assume that rational firms will first decide whether or not to implement ICT platforms specifically for KM. By this, we assume that it is possible to implement $\mathrm{KM}$ with the existing basic information system if firms decide not to implement ICT platforms that are specifically designed as a part of a KM system. Then the firm will decide whether or not to adopt a monetary incentive system for rewarding the sharing of knowledge. The two decisions are considered the basic strategies for encouraging knowledge-sharing.

Regarding the information technology infrastructure of a firm, it is assumed that the firm has a basic database or information system that can store documented knowledge or lessons learned shared by employees. An ICT platform, however, is built upon an existing database or information system and is a custom-designed advanced system or platform for knowledge-sharing, processing and retrieving. Also, it is assumed that ICT platforms have limited advantages in reducing the explicit costs of sharing.

Concerning the rewards for knowledge-sharing, the offering of monetary rewards is explicitly modelled as the firm's strategic choice. The non-monetary rewards are modelled as the intrinsic rewards received by the sharer, which are not directly associated with the firms' costs of encouraging sharing. In the model, we assume that the intrinsic rewards will be generated automatically through the social process in an organization and that firms will reasonably, but not particularly, emphasize improving the intrinsic rewards. Thus, in terms of the magnitude of the intrinsic rewards, it is assumed that the intrinsic rewards cannot compensate the costs of sharing if the costs are too high. In this model, we do not consider those rare occasions where the intrinsic rewards can be improved significantly to compensate high sharing costs.

\section{Model variables}

The model variables in this paper are the variables of the payoff functions of the players. We shall define these variables and make further modelling assumptions concerning these variables. Note that all model variables in this game are non-negative, real numbers.

\section{Variables regarding employees}

- $\gamma_{E}$ : Explicit costs of sharing knowledge

The explicit costs are defined as the time and efforts needed for an employee to share knowledge. The explicit costs of sharing are mostly related to the complexity of knowledge; for example, the explicit costs of sharing a journal article are low but the costs are high for sharing lessons learned from a project. Although the explicit sharing costs can also be affected by other factors, such as how busy the employee is, we shall simplify the model by focusing on the complexity of knowledge.

- $\gamma_{I}$ : Implicit costs of sharing knowledge When an individual shares his/her knowledge, the sharing may incur hidden costs due to the fact that the individual's competitiveness and uniqueness in a firm may be hampered after sharing their unique knowledge. The hidden costs of sharing are conceptualized as the 'implicit costs' and the magnitude of such costs for an employee mostly depends on the uniqueness to an employee and/or the importance of the knowledge within a firm.

- $s$ : Intrinsic rewards for sharing knowledge The 'intrinsic rewards' are non-monetary rewards. As individuals share their knowledge in organizations, they may get positive feedback from their 
colleagues, feel good about helping others or being appreciated or build up professional reputation or authority in a work group or firm.

- $w$ : Extrinsic rewards for sharing knowledge Offering extrinsic rewards such as monetary rewards to the sharers is a common approach for a firm to promote knowledge sharing. For the firm, if an employee's knowledge, once shared, is valuable to the firm, yet the costs of sharing are too high for the employee, the organization may promote the sharing through offering monetary rewards. The forms of rewards can also be designed to be contingent on the performance of the task; e.g. profit-sharing, gain-sharing, stock ownership, etc. (Bartol and Srivastava, 2002). Since the monetary rewards are to compensate the high costs of sharing, the monetary rewards can be significant if the costs of sharing are high.

\section{Variables regarding firms}

It is assumed that firms will devise certain strategies for better knowledge-sharing, specifically, the technologies and the incentive systems, characterized by the following parameters.

\section{- $c_{P}$ : Costs for ICT platforms}

The costs for custom-designed ICT platforms and platform implementations are usually accounted for as the major costs of $\mathrm{KM}$ and are often considered a costly investment.

- $c_{R}$ : Firm's costs for providing monetary rewards $c_{R}$ is the sum of the monetary rewards offered to individuals who share their knowledge.

- $\pi$ : Benefits of shared knowledge for a firm without ICT platforms

The benefits of shared knowledge can be considered the better or improved competitive advantages due to other employees' learning of the shared knowledge, the major reason for promoting $\mathrm{KM}$. Although these benefits are difficult to be precisely quantified, we assume that, in practice, they can be either qualitatively or quantitatively evaluated so as to compare with the costs for KM.

- $d$ : the demand for the shared knowledge

$d$ is a coefficient that multiplies $\pi$ and measures the demand of a firm for the shared knowledge. $d$ is conceptualized as the scale effect because of the knowledge dissemination advantages from having ICT platforms. $d$ is greater than or equal to one by definition. Thus, $d \pi$ will represent the benefits for the firm with ICT platforms. The demand for certain knowledge will be high when there are economies of scale, creating greater advantages of knowledge dissemination and utilization. Such economies of scale can be associated with many factors, such as the size of the firm and how fundamental the knowledge is. Alternatively, the demand for the knowledge can also be evaluated by the demand for fast response or for soliciting help from a large number of people.

\section{The game tree}

We assume that the knowledge-sharing game is a dynamic game with complete information, where firms provide the environment for knowledge-sharing and then employees decide to share or not. Figure 2 shows the game tree of knowledge-sharing dynamics. As shown, there are two players in the game, the employee and the firm. For the firm, there are two types of nodes. At the first node, the firm decides whether or not to implement ICT platforms by incurring the platform costs; thus, the choices at this first node are 'ICT platform' and 'No ICT platform'. At the firm's second node, the firm decides whether or not to reward the employees for knowledge-sharing, and the choices are 'Rewards' and 'No rewards'. At the employee's turn, the employee can decide to either 'Share' or 'Not share'.

The payoffs of the players are modelled as shown in Figure 2. For example, for the equilibrium following the path: Firm [ICT platform, Rewards] and Employee [Share] in the game tree, the payoffs for the firm are $d \pi-c_{P}-c_{R}$ and the payoffs for the employee are $s+w-\gamma_{E}-\gamma_{I}$. Following the same reasoning, the rest of the payoff profiles in the game tree shown in Figure 2 can be obtained.

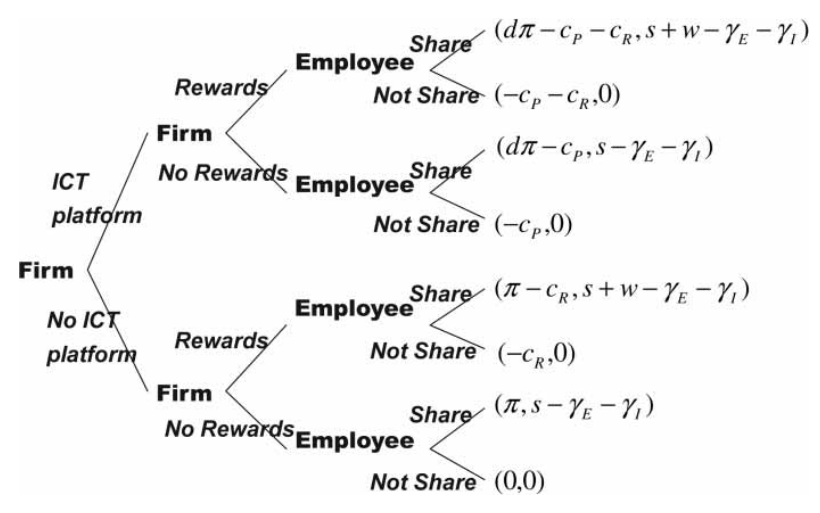

Figure 2. The game tree of knowledge-sharing dynamics

\section{Equilibrium solutions and their implications for knowledge-sharing}

By solving the game tree backward recursively, we will obtain eight possible game equilibriums and their corresponding equilibrium conditions. These equilibriums 
will follow five equilibrium paths, organized as five different cases where each case represents a unique combination of the decisions of the firm and the employee. Note that an equilibrium path is the path leading from the first node to the last node followed by possible equilibriums, whereas an equilibrium is a profile of players' strategies, specifying each player's action if the player is called upon to move in the game tree. It is possible that one equilibrium path will be followed by multiple equilibriums. Figure 3 shows the five equilibrium paths indicated by Cases I-V and the equilibriums following each path, indicated by \#1-8. In what follows, we will derive the conditions of the eight possible equilibrium solutions and discuss the implications from these conditions for knowledge-sharing strategies. The eight equilibriums are depicted by the eight game trees shown in Figure 4(a)-(h), where the bold lines in the game trees indicate each player's strategy in equilibrium.

\section{Case I: Firm [No ICT platform, No rewards]/Employee} [Share]

In this study, Firm [No ICT platform, No rewards]/ Employee [Share] denotes 'No ICT platform' and 'No rewards' for the firm and 'Share' for the employee. Each case in this section represents an equilibrium path, which can be followed by multiple subgame perfect Nash equilibriums. In this paper, the equilibrium in a game tree is indicated by a profile of bold lines following each node in a game tree, where the set of bold lines of each player represents that player's equilibrium strategy. There is one equilibrium for Case $\mathrm{I}$, as shown by equilibrium \#1 in Figure 4(a). Following the backward induction, the nodes for the employee should be solved first. In Case I, we consider the situation that the employee will share no matter what the firm's move is. For the employee at every node to be willing

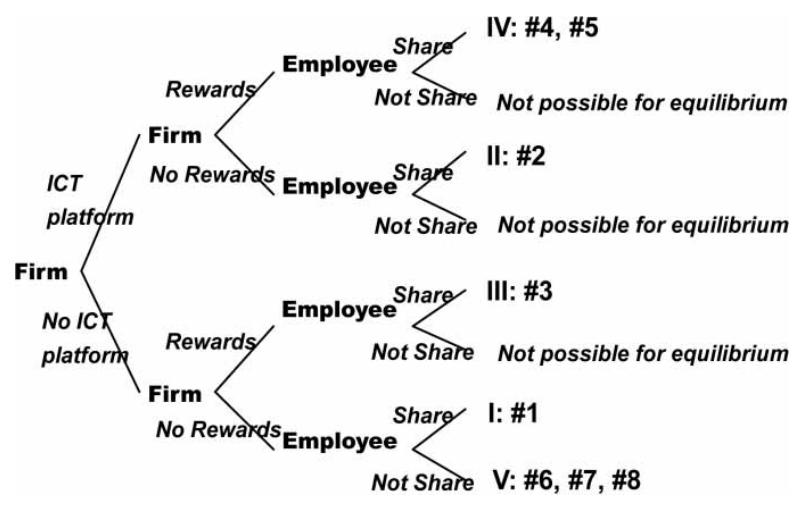

Figure 3 The five equilibrium paths and the associated equilibriums to share, the conditions for positive payoffs, $s-\gamma_{E}-$ $\gamma_{I}>0$ and $s+w-\gamma_{E}-\gamma_{I}>0$, must be satisfied. Note that since $s+w-\gamma_{E}-\gamma_{I}>0$ is implied by $s-$ $\gamma_{E}-\gamma_{I}>0$ given that the model variables are nonnegative, the only condition needed for the employee to share is $s-\gamma_{E}-\gamma_{I}>0$, condition (1). Next, we solve the firm's node for 'Rewards' or 'Not rewards'. Note that since the employee will share no matter if there are rewards or not given condition (1), the payoffs of choosing 'No rewards' will strictly dominate the payoffs of choosing 'Rewards'. Thus, the firm will choose 'No rewards' without imposing additional conditions. Lastly, we solve the firm's node for 'ICT platform' or 'No ICT platform' by comparing their respective payoffs, $d \pi-c_{P}$ and $\pi$, given Firm [No rewards] and Employee [Share]. We obtain that the firm will choose 'No ICT platform' if $\pi>d \pi-c_{P}$, rewritten as condition $2,(d-1) \pi<c_{P}$. To summarize, when conditions (1) and (2) are satisfied, equilibrium \#1 will be the equilibrium following the equilibrium path Firm [No ICT platform, No rewards]/Employee [Share]. Note that since 'No rewards' for firm dominates 'Rewards' given that the employee will share without rewards, the equilibrium following the path in Case $\mathrm{I}$ is unique.

$$
\begin{gathered}
s-\gamma_{E}-\gamma_{I}>0 \\
(d-1) \pi<c_{P}
\end{gathered}
$$

\section{Remarks}

Condition (1) signifies that the explicit costs of sharing knowledge, $\gamma_{E}$, and the implicit costs of sharing knowledge, $\gamma_{I}$, are relatively small compared with the intrinsic rewards from sharing, $s$. We shall use 'simple' for the characterization of knowledge that satisfies condition (1), meaning that it is easy and not costly for the employee to share this type of knowledge. The most probable situations for condition (2) are either that $\pi \rightarrow$ 0 (zero value to the firm) or that both $\pi$ and $d$ are small (low value and low demand), under which the investment in ICT platforms cannot be justified. In this study, we shall use 'low value to the firm' for the characterization of knowledge with low $\pi$. For knowledge with low $d$, it is characterized as knowledge with 'low demand' in the firm. As a result, knowledge satisfying conditions (1) and (2) are characterized by: 'simple', 'low value' and 'low demand'. We shall name knowledge of this type as 'trivial knowledge', implying that the knowledge is simple and irrelevant and can hardly be considered knowledge for the firm. The detailed discussion of knowledge taxonomy and associated firm strategies implied by game equilibriums will be 

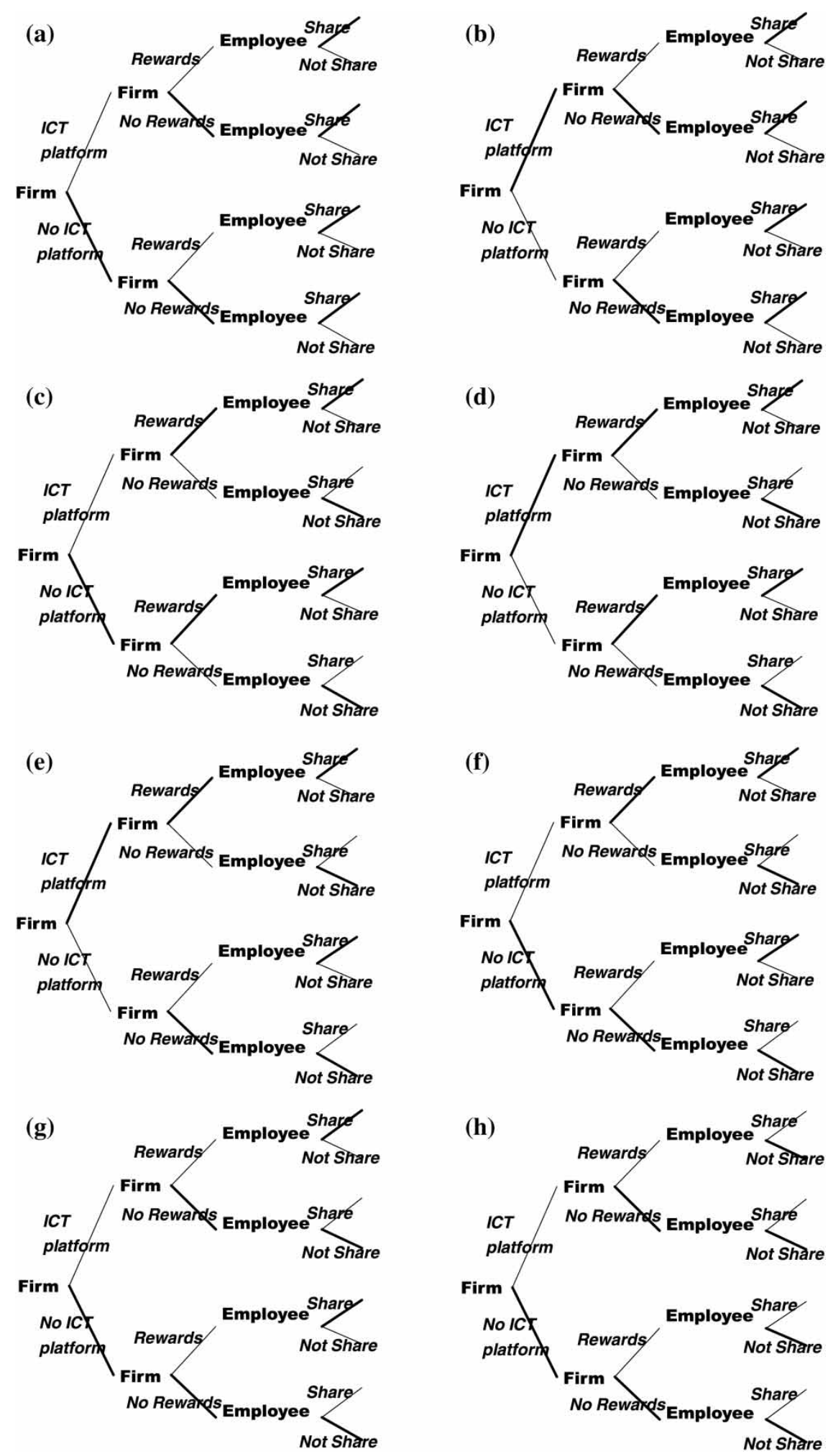

Figure 4 (a) Equilibrium \#1, (b) equilibrium \#2, (c) equilibrium \#3, (d) equilibrium \#4, (e) equilibrium \#5, (f) equilibrium $\# 6$, (g) equilibrium \#7, (h) equilibrium \#8 
presented in 'Model-implied knowledge taxonomy, hypotheses and knowledge-sharing strategies' section.

\section{Case II: Firm [ICT platform, No rewards]/Employee}

[Share]

Compared with Case I, the equilibrium path differs in the firm's decision on ICT platforms. For the firm to choose 'ICT platform', the payoffs of having 'ICT platform' have to be greater than that of 'No ICT platform', i.e. $d \pi-c_{P}>\pi$, which is rewritten as condition (3), $(d-1) \pi>c_{P}$. Thus, when conditions (1) and (3) are satisfied, equilibrium \#2 as shown in Figure 4(b) will be the equilibrium following the equilibrium path: Firm [ICT platform, No rewards]/Employee [Share]. Note that the equilibrium for the path in Case II is also unique for the same reason as in Case I:

$$
(d-1) \pi>c_{P} .
$$

\section{Remarks}

Given that the knowledge is 'simple' as indicated by condition (1), the most probable situation satisfying condition (3) is that the knowledge is 'low value' but with 'high demand'. In other words, the benefits to the firm from disseminating low value knowledge through ICT platforms are large enough to justify the platform costs. Since the demand of such simple knowledge is high enough to create high overall values to the firm and to justify the costs of ICT platforms, we shall name such knowledge as 'high demand simple knowledge'.

\section{Case III: Firm [No ICT platform, Rewards]/Employee [Share]}

Again, the Employee's node is solved first. Different from Cases I and II, here we start with the situation that the employee will share only when there are rewards for sharing. This scenario implies that the payoffs for sharing under 'No rewards', $s-\gamma_{E}-\gamma_{I}$, are negative but the payoffs for sharing under 'Rewards', $s+w-\gamma_{E}-\gamma_{I}$, are positive. Therefore, conditions (4) and (5) have to be satisfied in Case III. Next, we need to find the conditions for the firm to choose 'Rewards'. Two situations need to be considered. First, under 'No ICT platform', given conditions (4) and (5), the firm will choose 'Rewards' if the payoffs $\pi-c_{R}$ due to knowledge-sharing under 'Rewards' are greater than the payoffs due to the nonsharing of knowledge under 'No rewards'. Thus, condition (6), $\pi-c_{R}>0$, has to be satisfied for the firm to choose 'Rewards' under 'No ICT platform'. Second, under 'ICT platform', the firm will choose
'Rewards' if the payoffs $d \pi-c_{P}-c_{R}$ due to knowledge sharing under 'Rewards' are greater than the payoffs $-c_{P}$ due to the non-sharing of knowledge under 'No rewards'. As a result, the firm will choose 'Rewards' under 'ICT platform' if $d \pi-c_{P}-c_{R}>-c_{P}$, or rewritten as condition (7), $d \pi-c_{R}>0$. However, since $d \geq 1$, condition (7) can be implied by condition (6). We shall use $(7 \leftarrow 6)$ to denote that condition (7) is implied by (6). Lastly, the firm will choose 'No ICT platform' if $\pi-c_{R}>d \pi-c_{P}-c_{R}$, or rewritten as condition (2), is satisfied. Thus, when conditions (2), (4), (5), (6) and $(7 \leftarrow 6)$ are satisfied, equilibrium \#3 will be the equilibrium following the equilibrium path Firm [No ICT platform, Rewards]/Employee [Share]. Note that condition $(7 \leftarrow 6)$, although it can be omitted from the equilibrium conditions, remains in the required conditions for the purpose of future reference in case that this equilibrium is modified to become another equilibrium, as shown in the next case.

$$
\begin{gathered}
s-\gamma_{E}-\gamma_{I}<0 \\
s+w-\gamma_{E}-\gamma_{I}>0 \\
\pi-c_{R}>0 \\
d \pi-c_{R}>0 .
\end{gathered}
$$

Another possible equilibrium that may follow the equilibrium path in Case III is by changing equilibrium \#3 to 'No rewards' under 'ICT platform', which requires $d \pi-c_{P}-c_{R}<-c_{P}$, or rewritten as $d \pi-c_{R}<0$. However, $d \pi-c_{R}<0$ will contradict with condition (6), $\pi-c_{R}>0$, required for this possible equilibrium, as condition (6) implies $d \pi-c_{R}>0$. Therefore, this equilibrium cannot exist.

\section{Remarks}

As indicated by conditions (4) and (5), knowledgesharing by an employee may be costly such that the employee will share the knowledge only when there are sufficient extrinsic rewards from the firm to justify the costs of sharing. Knowledge characterized by conditions (4) and (5) shall be called 'complex/unique knowledge', which is complex in terms of high explicit costs of sharing and/or unique in terms of high implicit costs of sharing. Condition (6) indicates that the benefits to the firm due to knowledge-sharing without ICT platforms are higher than the costs of rewards. Since the rewards must compensate for the high costs of sharing so as to motivate the sharing, the benefits to the firm, $\pi$, can be considered high. Lastly, given $\pi$ is high, condition (2) for this equilibrium, $(d-1) \pi<c_{P}$, implies that the demand for the knowledge, $d$, must be 
low, such that ICT platforms cannot be justified, even though $\pi$ is high. Knowledge of this type is very important to a firm in terms of sustaining or improving the competitive advantages of the firm, but only a few employees in a firm need to learn or obtain such knowledge. As a result, knowledge of this type in Case III can be characterized by 'complex/unique', 'high value' and 'low demand'. We shall name this type of knowledge as 'low demand core knowledge'.

\section{Case IV: Firm [ICT platform, Rewards]/Employee [Share]}

Compared with Case III, the only difference in Case IV is that the payoffs of having 'ICT platform' have to be greater than that of 'No ICT platform', i.e. $d \pi-c_{P} \geq$ $\pi$ or rewritten as condition (3). Thus, when conditions (3) $-(6)$ and $(7 \leftarrow 6)$ are satisfied, equilibrium \#4 will be the equilibrium following the path in this case.

Another equilibrium following the path in this case is obtained by changing equilibrium \#4 to 'No Rewards' under 'No ICT platform', which requires condition (8), $\pi-c_{R}<0$, to replace condition (6). Since condition (6) is not required in this equilibrium, condition $(7 \leftarrow 6)$ in equilibrium \#4 will become explicit as condition (7) for this equilibrium. In addition, for the firm to choose 'ICT platform', it requires condition (9), $d \pi-c_{P}-c_{R}>0$, to replace condition (3). However, because of condition (9), condition (7) is again implied and expressed as $(7 \leftarrow 9)$. As a result, when conditions (4), (5), $(7 \leftarrow 9),(8)$ and (9) are satisfied, equilibrium \#5 will be the equilibrium following the path Firm [ICT platform, Rewards]/Employee [Share].

$$
\begin{gathered}
\pi-c_{R}<0 \\
d \pi-c_{P}-c_{R}>0 .
\end{gathered}
$$

\section{Remarks}

Since the conditions of equilibrium \#4 are obtained by substituting condition (3) for condition (2) in equilibrium \#3, it can be inferred that the demand for the knowledge in the firm is medium to high. Therefore, knowledge shared in this equilibrium can be characterized by 'complex/unique', 'high value' and 'medium to high demand'. Knowledge of this type is essential to the competitive advantages of a firm and is demanded by many employees.

On the other hand, knowledge shared in equilibrium \#5 is different from that in equilibrium \#4 in terms of its value and demand. Condition (8) shows that if there are no ICT platforms, the value of shared knowledge cannot justify the costs for rewarding the sharing. Given that the knowledge here is 'complex/unique', the value of the knowledge is most likely at the medium level. However, since the demand for the knowledge is high as indicated by condition (9), it is worth for the firm to reward the sharing and disseminate such knowledge to many employees in the firm. Therefore, knowledge of this type can be characterized by 'complex/unique,' 'medium value' and 'high demand'.

Since the value and demand of the knowledge shared in the two equilibriums in Case IV are at least at the medium level, we shall name this type of knowledge as 'high demand core knowledge'.

\section{Case V: Firm [No ICT platform, No rewards]/Employee [No share]}

There are three equilibriums (\#6-8) that will follow the equilibrium path in Case V. Equilibrium \#6 is obtained by changing equilibrium $\# 5$ to choosing 'No ICT platform', which requires condition (10), $d \pi-c_{P}-c_{R}<0$, to replace condition (9). Note that since condition (9) in equilibrium \#5 is removed, condition $(7 \leftarrow 9)$ will become explicit in this equilibrium as condition (7). Thus, equilibrium \#6 requires conditions (4), (5), (7), (8) and (10) to be satisfied.

$$
d \pi-c_{P}-c_{R}<0 .
$$

Equilibrium \#7 is obtained by changing equilibrium \#6 to 'No rewards' under 'ICT platform', which requires $d \pi-c_{P}-c_{R}<-c_{P}$, or rewritten as condition (11), to replace condition (7). Because of condition (11), condition (8) from equilibrium \#6 will be implied by (11) and expressed as $(8 \leftarrow 11)$. Next, for the firm to choose 'No ICT platform', no additional conditions are needed because the payoffs for choosing 'ICT platform', $-c_{P}$, are negative. As a result, equilibrium \#7 requires conditions (4), (5), $(8 \leftarrow 11)$ and (11) to be satisfied.

$$
d \pi-c_{R}<0
$$

Equilibrium \#8 is obtained when condition (12), $s+w-\gamma_{E}-\gamma_{I}<0$, is satisfied, under which the employee will not share in any cases. No additional conditions are required for this equilibrium because, given that the employee will not share, any investments in ICT platforms will yield negative payoffs.

$$
s+w-\gamma_{E}-\gamma_{I}<0
$$


Remarks

Conditions (7), (8) and (10) in equilibrium \#6 indicate that the value of knowledge is most likely at the medium level, not able to justify the costs of rewards, and the demand is most likely at a medium level, enough to justify rewards but not high enough to justify the combined use of ICT platforms and rewards. Condition (11) in equilibrium \#7 also indicates that the knowledge has medium value and low demand. As a result, no particular actions will be taken by firms to motivate the sharing of this type of knowledge and the employee will not share because of the high costs of sharing.

Knowledge in equilibrium \#6 and that in equilibrium \#7 are both characterized by 'complex/unique', 'medium value' and 'low to medium demand'. We shall name this type of knowledge as 'special knowledge', meaning that the value of the knowledge is not high enough to be rewarded because the knowledge is so specialized that only a few employees demand to learn or obtain such knowledge.

Condition (12) in equilibrium \#8 signifies that it is mostly likely that the knowledge is 'unique/complex', but the value of the knowledge is too low to justify any monetary rewards. Knowledge of this type is named as 'spurious knowledge', meaning that although it is costly for the employee to share such knowledge, the knowledge contributes very low value to the firm.

\section{Model-implied knowledge taxonomy, hypotheses and knowledge-sharing strategies}

\section{New knowledge taxonomy and the associated knowledge-sharing strategies}

Six types of knowledge are defined as shown in Table 1 based on the game equilibrium conditions and the knowledge characteristics implied by these conditions. Table 1 also shows firms' optimal knowledge-sharing strategies for each type of knowledge. The six types of knowledge are characterized by three dimensions: (1) the complexity or uniqueness of the knowledge associated with the costs of sharing, $\gamma_{E}$ and $\gamma_{I}$; (2) the value to the firm if the knowledge is shared in a firm without ICT platforms, expressed by $\pi$ and (3) the demand for the knowledge in a firm, $d$. Knowledge-sharing strategies associated with each type of knowledge are proposed and discussed if necessary. These strategies are mainly implied by the characteristics defining each type of knowledge.

\section{Trivial knowledge}

Trivial knowledge is characterized as simple, low value and in low demand. Forwarding an unimportant article or offering low quality, simple suggestions are

Table 1 Knowledge characteristics, taxonomy and equilibrium decisions

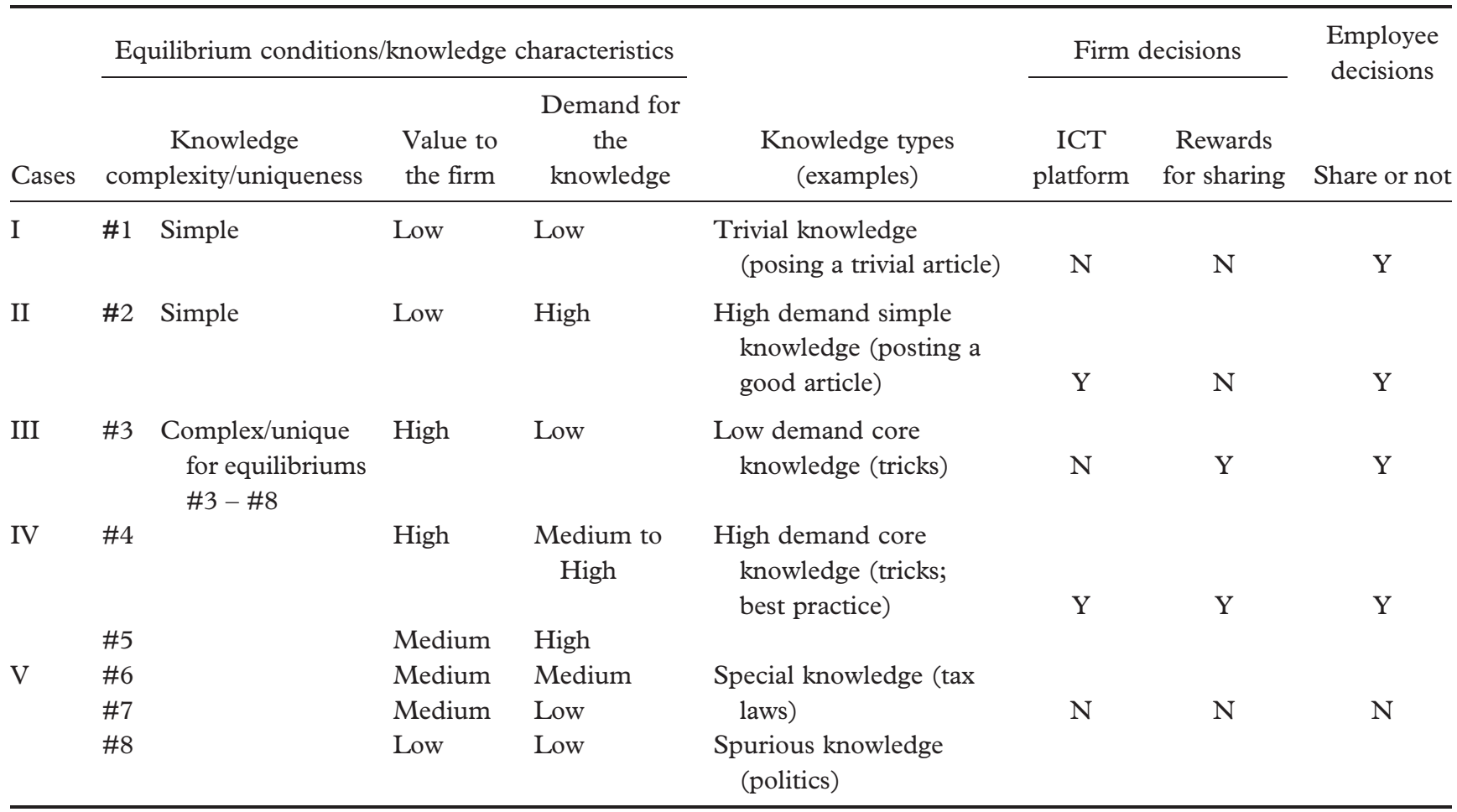

Note: N, no; Y, yes. 
examples of the sharing of trivial knowledge. Strategies implied by the knowledge characteristics and equilibrium results include the following:

- The firm should not provide either ICT platforms or rewards for trivial knowledge.

- Since trivial knowledge contributes little to the firm and can pollute knowledge bases and increase the costs of knowledge screening or searching, strategies for promoting knowledge-sharing should avoid or discourage the sharing of trivial knowledge.

- Employees under the pressure of demonstrating their willingness to share may tend to share trivial knowledge; thus, the evaluation of sharing performance should not focus mainly on sharing activities.

\section{High demand simple knowledge}

High demand simple knowledge is characterized as simple, low value and in high demand. Simple knowledge such as a good article from a journal can have high demand when there are economies of scale, creating advantages in knowledge dissemination and utilization. In particular, there may be valuable synergy in knowledge creation processes when a large amount of simple knowledge is shared or when a specific, simple knowledge is shared within a large organization. High demand can also be regarded as the demand for fast response or for soliciting help from a large number of people. For example, employees can request help or quick answers online from a large community of practice $(\mathrm{CoP})$ and expect to receive simple but timely and vital information for resolving a problem. The implied strategies for knowledge-sharing may include the following.

- Although no rewards are needed to encourage the sharing of such knowledge, ICT platforms can be justified by a firm's high demand of such knowledge.

- High demand simple knowledge can be important, targeted knowledge to be managed in KM.

- CoPs focusing on informal communications and social interactions are an important platform for sharing high demand simple knowledge, because simple knowledge fits informal communication and social interactions in CoPs will offer higher intrinsic rewards.

\section{Low demand core knowledge}

Low demand core knowledge is characterized as complex/unique, high value and in low demand. The firm size is an obvious reason for the low demand of core knowledge. There are other possible reasons for the low demand of core knowledge. For example, the low demand may be caused by the fact that the knowledge is highly tacit and can only be learned by face-to-face interaction or through the mentorapprentice relationship. It is also possible that the knowledge is critical only to a few employees of a certain group such as top managers or subsidiary managers. Extrinsic rewards are required for encouraging the sharing of high value core knowledge. The extrinsic rewards required can be broadly defined to include other forms of rewards, such as career promotion, salary raise, bonus or the high-performance evaluation of an employee. In a firm, this type of knowledge may often be possessed by senior managers or departmental managers, and as we have observed in practice, these managers are often willing to share their knowledge with their subordinates or apprentices in return for the better performance of the departments/sectors and the better chance for promotion. This knowledge-sharing process is implicit in the sense that the sharing is embedded in and encouraged by the organizational control and incentive system, even when there are no explicit implementations of $\mathrm{KM}$ in the firm. The implied strategies for knowledgesharing may include the following.

- Although knowledge of this type is essential to firms' competitive advantages and deserves rewards for encouraging the sharing, ICT platforms are not necessary because only a few people need to or can learn such knowledge.

- Extrinsic rewards provided by firms will be needed to encourage the sharing of this type of knowledge.

- In a firm, this type of knowledge may often be possessed by senior managers or departmental managers. In this case, the KM should focus more on the organizational control and incentive system that promotes higher efforts, including sharing and coaching, devoted by managers, instead of documentation of knowledge or platform implementation.

\section{High demand core knowledge}

High demand core knowledge is characterized as complex/unique, medium to high value and medium to high demand. Firms are suggested to offer rewards and establish ICT platforms when managing such knowledge. Other than large firms, firms characterized by high task repetitiveness can also generate high demand for the knowledge that may improve the performance of many of these repetitive tasks. For 
example, in a large firm, if the knowledge is associated with the improvement of certain critical/basic skills of most employees in the firm, even if the benefits of skill improvement are not significant for one employee, the cumulative benefits due to better skilled employees can become significant. In this regard, tricks, best practices and lessons learned are examples of such knowledge. The implied strategies for knowledge-sharing may include the following.

- This type of knowledge should be the most important target of the modern KM implementation of firms and deserve the investments in both ICT platforms and rewards.

- CoPs focusing on informal communications and social interactions are also important platforms for sharing high demand core knowledge, since that knowledge with low explicit sharing costs fits informal communication and that social interactions will offer higher intrinsic rewards to reinforce the higher sharing willingness.

- Formal systems for evaluating sharing performance should focus mainly on the sharing of high value and high demand knowledge, instead of the sharing activities or behaviours. The evaluation process that involves the evaluation by those who receive the knowledge is consistent with the focus on the high value and high demand criteria.

\section{Special knowledge}

Special knowledge is characterized as complex/unique, medium value and medium to low demand. For example, knowledge concerning a firm's information system design, detailed accounting principles, tax laws and knowledge from the CEO for strategic decisionmaking can be considered special knowledge, because they are more difficult to be shared, have limited marginal contributions due to the sharing and with very low demand for the knowledge. The demand is so low that in some cases the owners of the knowledge are the only ones in a firm who need such knowledge. The implied strategies for knowledge-sharing may include the following.

- According to the equilibrium, monetary rewards are needed to encourage the sharing of special knowledge due to the knowledge complexity/ uniqueness; but it is not economical for firms to provide such incentives because very few people need to have such knowledge. As a result, the best strategy for firms is to have no ICT platforms and no rewards and to let the knowledge be kept to these specialists.

\section{Spurious knowledge}

Lastly, spurious knowledge is characterized as complex/ unique, low value and in low demand. Outdated knowledge, irrelevant knowledge and textbook-type knowledge shared by new employees are examples of spurious knowledge. The term 'spurious' implies that this type of knowledge can be mistreated as valuable knowledge because the explicit costs of sharing spurious knowledge are high. The implied strategies for knowledge-sharing may include the following.

- The sharing of spurious knowledge requires significant efforts from employees; thus, the sharing of such knowledge should be discouraged by firms.

- Neither ICT platforms nor rewards should be provided.

- Similar to trivial knowledge, if monetary rewards are not properly designed to reward the sharing of core knowledge, the sharing of spurious knowledge can be wrongly incentivized.

\section{Hypotheses and the associated knowledge- sharing strategies}

Three testable hypotheses regarding knowledge-sharing strategies are proposed based on the results of the game theory analysis. These hypotheses can be empirically tested in future studies so as to evaluate the contingency theory of knowledge-sharing developed in this study. Different from the strategies associated with a particular type of knowledge, the strategies proposed in this section are associated with all types of knowledge and are in the context of the implementation of KM.

Hypothesis 1: The greater the number of employees who possess 'high demand simple knowledge' and/ or 'high demand core knowledge', the higher the performance of KM with ICT platforms.

This hypothesis can be drawn from the game theory analysis summarized in Table 1 . The implied strategy for knowledge-sharing is as follows.

- In considering the adoption of ICT platforms, firms should evaluate how large is the number or proportion of employees who possess 'high demand simple knowledge' and/or 'high demand core knowledge'.

Hypothesis 2: The greater the number of employees who possess 'low demand core knowledge' and/or 'high demand core knowledge', the higher the performance of KM with monetary rewards.

This hypothesis is due to the game analysis result that core knowledge deserves the rewards for encouraging 
the sharing of knowledge. The implied strategies for knowledge-sharing may include the following.

- The rewards should be used to reward those who share the most wanted or valuable knowledge, instead of those who share the most.

- The most wanted knowledge to be rewarded should be either the 'low demand core knowledge' or 'high demand core knowledge'. However, when there are ICT platforms, the high demand core knowledge should deserve highest rewards because of the greater overall benefits from the high demand.

- Rewarding the sharing behaviour without proper differentiation of the contents will encourage the sharing of unwanted knowledge, which will have many disadvantages such as the pollution of knowledge bases, the waste of efforts in over-sharing and the consequent reduction of productivity and fewer rewards left for motivating the sharing of desired knowledge.

Hypothesis 3: For firms with a satisfactory $\mathrm{KM}$ implementation, the employees who possess spurious knowledge or special knowledge will have the lowest willingness for sharing and the employees who possess high demand simple knowledge or high demand core knowledge will have the highest willingness for sharing.

This hypothesis is due to the game analysis result that firms should not motivate the employees by rewards to share trivial knowledge, spurious knowledge and special knowledge. However, since the sharing of trivial knowledge is motivated intrinsically, the employees who possess spurious knowledge and special knowledge will have the lowest willingness to share. The implied strategies for knowledge-sharing may include the following.

- For firms that require employees to fulfill a certain level of knowledge-sharing, the required levels of sharing for those employees who may possess trivial knowledge, spurious knowledge or special knowledge, such as new employees or supporting staff should be lower than that for other employees so as to prevent the sharing of unwanted knowledge.

\section{Conclusions}

From the RBV, KM can be considered one of the most critical processes for business success in today's intensively competitive environment. However, for those who practice $\mathrm{KM}$, the major effort in $\mathrm{KM}$ implementation is to develop the ICT platforms.
Unfortunately, many KM attempts died with their costly and advanced high-tech ICT platforms. In fact, it is becoming clear that KM cannot be successful without appropriate strategies for encouraging the sharing of knowledge, because people in organizations are not motivated to share valuable knowledge.

In this study, we develop a game-theoretic model for analysing knowledge-sharing dynamics and derive firm strategies for knowledge-sharing. Based on the results of game theory analysis, six types of knowledge are defined from the perspective of knowledge-sharing dynamics, namely, trivial knowledge, high demand simple knowledge, low demand core knowledge, high demand core knowledge, special knowledge and spurious knowledge. Each type of knowledge is characterized by different combinations of three knowledge characteristics: the knowledge complexity/uniqueness, value to the firm and demand for the knowledge. Strategies implied by the characteristics of the knowledge are proposed for each type of knowledge. Based on the new knowledge taxonomy, three empirically testable hypotheses were developed. The test of these hypotheses is left for future studies.

Based on the hypotheses, strategies for knowledgesharing concerning all types of knowledge and the implementation of KM are proposed. To summarize, in considering the adoption of ICT platforms, firms should evaluate how large is the number or proportion of employees who possess high demand simple knowledge and/or high demand core knowledge. In considering the offering of rewards, the most wanted knowledge to be rewarded should be either the low demand core knowledge or high demand core knowledge. However, when there are ICT platforms, the high demand core knowledge should deserve highest rewards because of the greater overall benefits from the high demand. As a result, firms should not expect that all their employees participate in sharing knowledge with the same enthusiasm. The expected levels of knowledge-sharing for those employees who may possess trivial knowledge, spurious knowledge or special knowledge, such as new employees or supporting staff, should be much lower than that for other employees so as to prevent the sharing of unwanted knowledge.

To conclude, this paper develops a contingency theory of knowledge-sharing strategies. From the perspective of knowledge-sharing dynamics, knowledgesharing strategies should aim to facilitate the sharing of valuable knowledge, instead of the sharing behaviour itself. The strategies for knowledge-sharing shall be formed contingent on the characteristics of knowledge. These characteristics of knowledge are also contingent on the interactions between the nature of knowledge, employees and the firm environments, such as firm scale and competitive advantages. Note that, although 
the offering of monetary rewards seems effective and simple, studies show that monetary rewards are less effective in promoting sustained knowledge-sharing attitudes and behaviours. Intrinsic rewards associated with social motivations may play a critical role in knowledge-sharing behaviours (Javernick-Will, 2011). For future studies, intrinsic rewards and their impacts on knowledge-sharing and associated strategies can be analysed by game theory modelling. Empirical studies can be conducted for the verification of the model developed in this study. Finally, knowledge owners, in terms of different classes of employees, of each type of knowledge can be identified and studied based on their behavioural characteristics in knowledge-sharing.

\section{Acknowledgements}

Financial support from National Science Council (Grant number: NSC 97-2221-E-002-204) of Taiwan are gratefully acknowledged.

\section{References}

Bartol, K.M. and Srivastara, A. (2002) Encouraging knowledge sharing: the role of organizational reward systems. Fournal of Leadership \& Organizational Studies, 9(1), 64-76.

Cabrera A. and Cabrera E.F. (2002) Knowledge-sharing dilemmas. Organization Studies, 23(5), 687-710.

Gibbons R. (1992) Game Theory for Applied Economists, Princeton University Press, Princeton, NJ.

Grant R.M. (1996) Prospering in dynamically competitive environments: organizational capability as knowledge integration. Organization Science, 7(4), 375-87.

Hendriks P. (1999) Why sharing knowledge? The influence of ICT on the motivation for knowledge sharing. Knowledge and Process Management, 6(2), 91-100.

Javernick-Will, A. (2011) Motivating knowledge sharing in engineering and construction organizations: the power of social motivations. ASCE fournal of Management in Engineering, under review.

Moon H.K. and Park M.S. (2002) Effective reward systems for knowledge sharing: facilitating knowledge flow at Samsung Life Insurance. Knowledge Management Review, $4(6), 22-5$. 
